Gefässchirurgie 2012 $17: 218-218$

DOI 10.1007/s00772-011-0974-3

Online publiziert: 14. März 2012

๑) Springer-Verlag 2012

\author{
K. Maier ${ }^{1}$ U.E. Klemp ${ }^{2} \cdot$ H. Böhner ${ }^{3}$ \\ ${ }^{1}$ Berlin \\ ${ }^{2}$ Klinik für Gefäß- und endovaskuläre Chirurgie, Phlebologie, Marienkrankenhaus, Soest \\ ${ }^{3}$ Chirurgische Klinik I, Katholisches Krankenhaus Dortmund-West, Dortmund
}

\title{
Leserbrief zur epiduralen Rückenmarkstimulation (SCS) als Therapieoption für selektierte pAVK-Patienten
}

\section{Korrespondenzadresse}

Springer-Verlag gewohnt bin. Unabhängig vom Inhalt scheint es schon bei der Zuordnung der Literatur Probleme gegeben zu haben. So kann der Leser keine „internationalen Leitlinien“ in den Literaturstellen 7, 8 und 10 finden, die zur - Tab. 2 gehören. Es handelt sich hierbei um kleine Studien oder Nachbeobachtungen. Die Empfehlungen der Gesellschaft für Anästhesie werden im Zusammenhang mit chronischen Gefäßerkrankungen zitiert (Literaturstelle 6) und so bleibt offen, ob die in $\bullet$ Tab. 2 aufgeführten Pausierungszeiten der Medikamente persönliche Empfehlungen sind oder von irgendeiner Literaturstelle bezogen wurden. Zitiert wird hierzu nichts, denn auch in den Empfehlungen der Gesellschaft für Anästhesie geht es nicht um SCS, sondern um Regionalanästhesien, was aus neurochirurgischer Sicht nicht übertragbar ist. Wenn solche Indikationen, Kontraindikationen oder Beschränkungen publiziert werden, sollten sie zumindest mit Literatur belegt werden.

Vielleicht haben Sie oder Ihre Reviewer die Möglichkeit, die Plausibilität solcher Zitate in der Zukunft besser zu überprüfen.

Mit freundlichen Grüßen

Klaus Maier

Dr. Klaus Maier

Pankower Allee 3

13409 Berlin

maierklaus80@yahoo.de

Die zu zitierenden Literaturstellen hätten 5,6 und 9 sein müssen.

Darüber hinaus kritisiert der Kollege die Tatsache, dass wir in Bezug auf die Limitation des Eingriffs durch Antikoagulanzien und Antiaggreganzien auf die Leitlinien der Anästhesiologischen Gesellschaften für rückenmarksnahe Narkosen zurückgegriffen haben. Da die Implantation eines SCS-Gerätes invasiver erscheint als eine normale rückenmarksnahe Narkose, halten wir diese Analogie für vertretbar.

Für die Autoren

Dr. U. Klemp

Dr. H. Böhner

\section{Korrespondezadresse}

\section{Dr. U.E. Klemp}

Klinik für Gefäß- und endovaskuläre Chirurgie

\section{Erwiderung}

\section{U.E. Klemp ${ }^{1}$, H. Böhner ${ }^{2}$}

${ }^{1}$ Klinik für Gefäß- und endovaskuläre Chirurgie,

Phlebologie, Marienkrankenhaus, Soest

${ }^{2}$ Chirurgische Klinik I, Katholisches

Krankenhaus Dortmund-West, Dortmund

Wir danken Herrn Dr. Maier für die Zusendung seines kritischen Kommentares.

Er weist zu Recht daraufhin, dass die zitierten Literaturstellen 7, 8 und 10 keine internationalen Leitlinien darstellen. Hier ist ein redaktioneller Fehler bei der $\mathrm{Zu}$ ordnung der Literaturstellen unterlaufen.
Widumgasse 5

59494 Soest

u.klemp@mkh-soest.de
Phlebologie, Marienkrankenhaus 